https://doi.org/10.15407/ujpe66.11.936

V.V. SKALOZUB, M.S. DMYTRIIEV

Oles Honchar Dnipro National University

(72, Gagarin Ave., Dnipro 49010, Ukraine;

e-mails: Skalozubv@daad-alumni.de,dmytrijev_m@ffeks.dnu.edu.ua)

\title{
ON DIRECT SEARCH FOR DARK MATTER IN SCATTERING PROCESSES WITHIN YUKAWA MODEL
}

\begin{abstract}
Nowadays, no dark matter candidates have been discovered. We consider two possible reasons for that, both related to the approach of on-peak resonance searching for. As is believed usually, a new particle suits the conditions that the ratio of the width to the mass is less than 1-3\% and a narrow-width approximation (NWA) is applicable to identify such type resonant peak in the invariant mass spectrum of the collision products. In the present paper, in the framework of a generalized Yukawa model, we find out the properties of the searched particle, when its width is larger than a maximal one expected during experiments, and, so, this state could be confused with a noise. We also ascertain the values of particle's parameters, when the NWA is not applicable and estimate the width value, when it happens. These estimations are relevant to interactions between the Standard model and dark matter particles. Such approach is focused on the role of couplings and mass values introduced in the model describing the interaction of visible and dark matters.
\end{abstract}

Keywords: dark matter, resonance, narrow-width approximation, mixing of fields.

\section{Introduction}

Nowadays, no particles-candidates for dark matter (DM) have been found yet. There is a long list of such particles entering different models relevant to various energy scales. In what follows, we consider why it could be so within the standard treating of scattering experiment data. It is usually assumed that the resonances of the unknown particles are narrow ones and their typical width $\Gamma$ is about $1-3 \%$ of the peak's mass $M$, so $\Gamma \ll M$. This assumption allows one to apply the NWA to detect the resonances in the total cross-section. In this approach, the interference between visible and dark particles could be neglected in the total cross-section. At the same time, there are many models beyond the Standard model (SM) present in the literature, which anticipate new particles to have wide resonances. Consequently, such signals can be confused with a noise, if they appear in experimental data. In the present paper, we analyze how the widths of new-physics peaks depend on masses and couplings introduced in some underlying model of the DM.

(c) V.V. SKALOZUB, M.S. DMYTRIIEV, 2021

936
We will carry out our investigation in the framework of a generalized Yukawa model. DM is represented there as the heavy Dirac fermion $\Psi$ and scalar $\chi$ fields. The visible matter is described as a light scalar field $\phi$ and the doublet of light fermion fields $\psi_{1}$ and $\psi_{2}$. Those fermion fields interact with bosons $\phi$ and $\chi$ through different Yukawa's couplings. Using this model, we obtain the width of a dark boson $\chi$ for certain values of the model parameters. Such approach is different from that used in some nonNWA peak investigations presented in, for instance, [1-3]. In these papers, the width of a new particle is adopted to be an arbitrary free parameter.

Nowadays, there exist numerous models of DM such as SUSY particles (neutralinos) [4], neutral $Z^{\prime}$ bosons [5-7], sterile neutrinos [8], etc. In these models, it is assumed that a DM candidate has a certain symmetry group or specified couplings to other fields. On the contrary, we consider all the parameters of DM as free, and do not limit our treatment by a certain gauge group. In addition, in what follows we do not consider the astrophysical limits on the couplings between the visible and dark sectors. These couplings affect the production and decay rates of DM and so its

ISSN 2071-0194. Ukr. J. Phys. 2021. Vol. 66, No. 11 
relic abundance in the Universe. Such problems could be investigated in the context of the energy distributions of the dark and visible particles. All these topics are left beyond the scope of the present paper.

Below, we are concentrating on the analysis of the particle parameter values for which the dark resonance does not satisfy the NWA bounds and becomes invisible in the direct search for it. Then we derive the necessary constraints on the properties of the DM sector.

The paper is organized as follows. In the following section, we introduce our model and discuss the mixing of scalar fields, which appears at the one-loop level. We also define the corresponding mixing angle. In Sect. 3, we consider the width of a dark particle resonance in the context of a chosen $s$-channel scattering process. Then we estimate the values of the parameters, when this width exceeds the NWA limits, and provide the limitations for the mixing angle. We summarize and discuss our results in the context of comparizons with a number of DM models in the last section.

\section{The Model}

We start with the Lagrangian

$\mathcal{L}=\frac{1}{2}\left[\left(\partial_{\mu} \phi\right)^{2}-\mu^{2} \phi^{2}\right]+\frac{1}{2}\left[\left(\partial_{\mu} \chi\right)^{2}-\Lambda^{2} \chi^{2}\right]-$

$-\lambda \phi^{4}-\rho \phi^{2} \chi^{2}-\xi \chi^{4}+$

$+\sum_{a=1 ; 2} \bar{\psi}_{a}\left(i \gamma^{\mu} \partial_{\mu}-g_{\phi} \phi-g_{\chi} \chi-m_{a}\right) \psi_{a}+$

$+\bar{\Psi}\left(i \gamma^{\mu} \partial_{\mu}-M-G_{\chi} \chi\right) \Psi$.

Dark fermions $\Psi$, having only the coupling $G_{\chi}$ to scalars in the dark sector, do not interact with visible bosons $\phi$. Due to the presence of both couplings $g_{\phi}$ and $g_{\chi}$, the scalar fields are mixed at the oneloop level, with the mixing angle $\theta_{\text {mix }}$. The value of $\theta_{\text {mix }}$ regulates the intensity of the interaction between the visible and dark sectors. In particular, this mixing expresses itself as the non-diagonal loop corrections coming from fermionic loops in the scalar two-point Green functions depicted in Fig. 1. We introduce the mixing angle as a $O(2)$-rotation, which diagonalizes the mass matrix of scalar fields at the zero momentum. This matrix emerges from the effective potential of bosons for constant fields $\phi$ and $\chi$. The total effective potential $V_{\text {eff }}$ consists of the tree-level part $V_{\text {eff }}^{\text {(tree) }}$ and a correction, which goes from Yukawa's

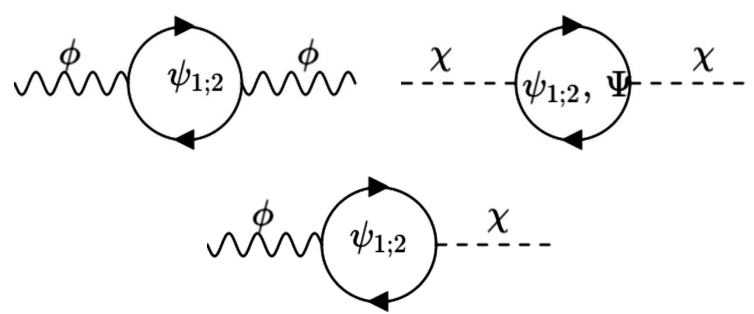

Fig. 1. Fermion loop corrections to the two-point Green functions $\left\langle 0\left|T \phi\left(x_{1}\right) \phi\left(x_{2}\right)\right| 0\right\rangle, \quad\left\langle 0\left|T \chi\left(x_{1}\right) \chi\left(x_{2}\right)\right| 0\right\rangle \quad$ and $\left\langle 0\left|T \phi\left(x_{1}\right) \chi\left(x_{2}\right)\right| 0\right\rangle$, respectively. Fermion fields are listed inside loops which they contribute to

interaction obtained after the integration over virtual fermions,

$V_{\mathrm{eff}}^{(\text {tree })}=\frac{1}{2} \mu^{2} \phi^{2}+\frac{1}{2} \Lambda^{2} \chi^{2}+\lambda \phi^{4}+\rho \phi^{2} \chi^{2}+\xi \chi^{4}$,

$V_{\text {eff }}=V_{\text {eff }}^{(\text {tree })}+\lim _{\epsilon \rightarrow 0} \frac{\kappa^{2 \epsilon}}{8 \pi^{2}} \int_{0}^{\infty} \frac{d s}{s^{3-\epsilon}} \times$

$\times\left\{\sum_{a=1 ; 2} e^{-s\left(m_{a}+g_{\phi} \phi+g_{\chi} \chi\right)^{2}}+e^{-s\left(M+G_{\chi} \chi\right)^{2}}\right\}=$

$=V_{\mathrm{eff}}^{(\text {tree })}-\frac{1}{16 \pi^{2}} \times$

$\times\left\{\sum_{a=1 ; 2}\left(m_{a}+g_{\phi} \phi+g_{\chi} \chi\right)^{4} \ln \frac{\left(m_{a}+g_{\phi} \phi+g_{\chi} \chi\right)^{2}}{\kappa^{2}}+\right.$

$\left.+\left(M+G_{\chi \chi}\right)^{4} \ln \frac{\left(M+G_{\chi} \chi\right)^{2}}{\kappa^{2}}\right\}+$

$+\frac{\Delta}{16 \pi^{2}}\left\{\sum_{a=1 ; 2}\left(m_{a}+g_{\phi} \phi+g_{\chi} \chi\right)^{4}+\left(M+G_{\chi} \chi\right)^{4}\right\}$,

$\Delta=\frac{1}{\epsilon}-\gamma_{E} \quad(\epsilon \rightarrow 0)$.

Here, $\gamma_{E}$ is the Euler-Mascheroni number, $\Delta$ is a divergent constant, and $\kappa$ is a renormalization point. We adopt $\kappa$ arbitrarily and account for the effects of the model couplings and masses, only. The integral in (2) involves fermionic corrections to the bosonic masses and the self-interaction constants $\lambda, \rho$, and $\xi$.

The components of the bosonic mass matrix $M_{a b}^{2}$ are given as the coefficients of the $V_{\text {eff }}$ Taylor's series expansion calculated at $\phi_{0}$ and $\chi_{0}$ :

$M_{11}^{2}=\left.\frac{\partial^{2} V_{\text {eff }}}{\partial \phi^{2}}\right|_{\phi_{0} ; \chi_{0}}, \quad M_{12}^{2}=\left.\frac{\partial^{2} V_{\text {eff }}}{\partial \phi \partial \chi}\right|_{\phi_{0} ; \chi_{0}}$,
$M_{22}^{2}=\left.\frac{\partial^{2} V_{\text {eff }}}{\partial \chi^{2}}\right|_{\phi_{0} ; \chi_{0}}$. 


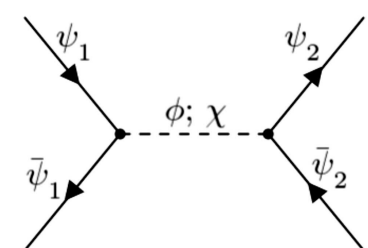

Fig. 2. Feynman diagram of the investigated process. Bold points denote the vertices calculated in the one-loop aproximation. Bold dashed line corresponds to the two-point Green function of a scalar doublet with the one-loop corrections

In what follows, we choose $\phi_{0}=0$ and $\chi_{0}=0$ that sets the renormalization point of bosonic masses. Hence, the mass matrix components are

$$
\begin{aligned}
& M_{11}^{2}=\mu^{2}-\frac{3 g_{\phi}^{2}}{4 \pi^{2}} F+\frac{3 g_{\phi}^{2}}{4 \pi^{2}}\left(m_{1}^{2}+m_{2}^{2}\right) \Delta, \\
& M_{12}^{2}=-\frac{3 g_{\phi} g_{\chi}}{4 \pi^{2}} F+\frac{3 g_{\phi} g_{\chi}}{4 \pi^{2}}\left(m_{1}^{2}+m_{2}^{2}\right) \Delta, \\
& M_{22}^{2}=\Lambda^{2}-\frac{3}{4 \pi^{2}}\left(g_{\chi}^{2} F+G_{\chi}^{2} M^{2} \ln \frac{M^{2}}{\kappa^{2}}\right)+ \\
& +\frac{3}{4 \pi^{2}}\left[g_{\chi}^{2}\left(m_{1}^{2}+m_{2}^{2}\right)+G_{\chi}^{2} M^{2}\right] \Delta, \\
& F=m_{1}^{2} \ln \frac{m_{1}^{2}}{\kappa^{2}}+m_{2}^{2} \ln \frac{m_{2}^{2}}{\kappa^{2}} .
\end{aligned}
$$

In these equalities, we do not account for the terms coming from the scalar self-interaction. This is because such terms are proportional to $\phi_{0}^{2}, \phi_{0} \chi_{0}$ or $\chi_{0}^{2}$. But since $\phi_{0}$ and $\chi_{0}$ are zeroes, their contributions vanish. The mass matrix components, accounting for the fermionic loop corrections, become divergent, and a mass renormalization is required. To do this, we add such counter-terms to $V_{\text {eff }}$ that the divergent parts proportional to $\Delta$ in (4) vanish ${ }^{1}$.

Having the angle $\theta_{\text {mix }}$, we rotate the scalar fields $\phi$ and $\chi$, turning them to the basis of "physical" states $\phi^{\prime}$ and $\chi^{\prime}$ :

$$
\left(\begin{array}{l}
\phi \\
\chi
\end{array}\right)=\left(\begin{array}{cc}
\cos \theta_{\text {mix }} & -\sin \theta_{\text {mix }} \\
\sin \theta_{\text {mix }} & \cos \theta_{\text {mix }}
\end{array}\right)\left(\begin{array}{l}
\phi^{\prime} \\
\chi^{\prime}
\end{array}\right) .
$$

\footnotetext{
1 To perform such renormalization, we use counter-terms $-\frac{1}{2} \delta \mu^{2} \phi^{(\text {ren }) 2}$ and $-\frac{1}{2} \delta \Lambda \chi^{(\text {ren }) 2}$, where $\phi^{(\text {ren })}$ and $\chi^{(\text {ren })}$ are fields rotated by the angle $\theta_{\text {ren }}$ with respect to $\phi$ and $\chi$. Hence, the renormalization procedure in our model consists of the $O(2)$-rotation and subtraction of divergent terms. The angle $\theta_{\text {ren }}$ is defined as follows:
}

$$
\tan 2 \theta_{\text {ren }}=\frac{2 g_{\phi} g_{\chi}}{g_{\phi}^{2}-g_{\chi}^{2}-G_{\chi}^{2} \frac{M^{2}}{m_{1}^{2}+m_{2}^{2}}} .
$$

We pick $\theta_{\text {mix }}$ requiring the non-diagonal term $M_{12}^{2}$ of the mass matrix to vanish for the rotated fields $\phi^{\prime}$ and $\chi^{\prime}$. It happens, when $\theta_{\text {mix }}$ is equal to

$$
\begin{aligned}
& \tan 2 \theta_{\text {mix }}=\frac{2 M_{12}^{2}}{M_{11}^{2}-M_{22}^{2}}=2 g_{\phi} g_{\chi} F\left[\frac{4 \pi^{2}}{3}\left(\Lambda^{2}-\mu^{2}\right)+\right. \\
& \left.+\left(g_{\phi}^{2}-g_{\chi}^{2}\right) F-G_{\chi}^{2} M^{2} \ln \frac{M^{2}}{\kappa^{2}}\right]^{-1} .
\end{aligned}
$$

In our model, the field mixing phenomenon appears starting from the one-loop level. Hence, we have that $\tan 2 \theta_{\text {mix }} \sim g_{\phi} g_{\chi}, \sin 2 \theta_{\text {mix }} \sim g_{\phi} g_{\chi}$ and $\cos 2 \theta_{\text {mix }} \sim 1$. Therefore, the sine and tangent of the $2 \theta_{\text {mix }}$ are quantities of the second order in the expansion series in the Yukawa couplings. Because of this, we omit, in what follows, such quantities as $g_{\phi}^{2} \tan 2 \theta_{\text {mix }}$, $g_{\phi} g_{\chi} \sin 2 \theta_{\text {mix }}$, etc., which correspond to the next-toleading corrections.

Moreover, the mixing angle roughly is proportional to $(\Lambda-\mu)^{-1}$. So, if one of the bosons is much heavier than another one, i.e., if $\mu \ll \Lambda$ or $\mu \gg \Lambda$, then $\theta_{\text {mix }}$ becomes very small. In this case, two particle resonances are located far from each other. As a result, the properties of the dark boson $\chi$ do not affect the mass or width of the visible particle $\phi$, and the dark matter remains invisible. Alternatively, if we put $g_{\chi} \ll g_{\phi}$, then $\theta_{\text {mix }}$ may be very small, and the dark matter signal will be indistinguishable from the background of visible particles. Thus, the weak interplay of two sectors in the model corresponds to small values of the mixing angle between the $\phi$ and $\chi$ states.

\section{Width of DM Particles}

Let us consider the scattering process shown in Fig. 2.

In this reaction, a pair of visible fermions $\psi_{1}$ and $\bar{\psi}_{1}$ annihilates into other visible fermion pair $\psi_{2}$ and $\bar{\psi}_{2}$. As an intermediate state, we have both $\phi$ and $\chi$ bosons. Besides the diagrams in Fig. 1, we consider the contributions coming from the one-loop vertex corrections arising from the diagrams such as that in Fig. 3. The matrix element of the process in Fig. 2 in the improved Born approximation has the following expression:

$$
\begin{aligned}
& i \mathcal{M}^{\text {(imp. Born) }}=i\left(\begin{array}{l}
g_{\phi}\left(1+\delta \Gamma_{\phi}^{(\text {fin })}\left(p_{3} ; p_{4}\right)\right) \\
g_{\chi}\left(1+\delta \Gamma_{\chi}^{(\text {fin })}\left(p_{3} ; p_{4}\right)\right)
\end{array}\right)^{T} \times \\
& \times\left(\begin{array}{cc}
p^{2}-\mu^{2}-\Pi_{\phi \phi}\left(p^{2}\right) & -\Pi_{\phi \chi}\left(p^{2}\right) \\
-\Pi_{\phi \chi}\left(p^{2}\right) & p^{2}-\Lambda^{2}-\Pi_{\chi \chi}\left(p^{2}\right)
\end{array}\right)^{-1} \times
\end{aligned}
$$

ISSN 2071-0194. Ukr. J. Phys. 2021. Vol. 66, No. 11 
$\times\left(\begin{array}{l}g_{\phi}\left(1+\delta \Gamma_{\phi}^{(\mathrm{fin})}\left(p_{1} ; p_{2}\right)\right) \\ g_{\chi}\left(1+\delta \Gamma_{\chi}^{(\mathrm{fin})}\left(p_{1} ; p_{2}\right)\right)\end{array}\right)$.

Here, $\delta \Gamma_{\phi}^{(\text {fin })}$ and $\delta \Gamma_{\chi}^{(\text {fin })}$ denote the renormalized loop corrections depicted in the diagram 3 , which we calculate numerically ${ }^{2} . \Pi_{\phi \phi}\left(p^{2}\right), \Pi_{\phi \chi}\left(p^{2}\right)$, and $\Pi_{\chi \chi}\left(p^{2}\right)$ are the renormalized components of the scalar field polarization operators. They comprise the contributions from the diagrams in Fig. 1 and those from the self-interaction of $\phi$ and $\chi$ shown in Fig. 4.

The scalar polarization operator components have been renormalized by using the following conditions:

$$
\begin{aligned}
& \Re \Pi_{\phi \phi}\left(p^{2}=\mu^{2}\right)=0, \quad \Re \Pi_{\chi \chi}\left(p^{2}=\Lambda^{2}\right)=0, \\
& \Re \Pi_{\phi \chi}\left(p^{2}=\kappa^{2}\right)=0 .
\end{aligned}
$$

Here, the diagonal terms $\Pi_{\phi \phi}\left(p^{2}\right)$ and $\Pi_{\chi \chi}\left(p^{2}\right)$ were renormalized on the mass shells of the corresponding bosons. For the non-diagonal component $\Pi_{\phi \chi}\left(p^{2}\right)$, an arbitrary renormalization point $\kappa^{2}$ was used. It is worth noting that, in the one-loop approximation, we would have to consider the box diagrams of the process in Fig. 2. However, the numerical simulations show that their contribution to the squared modulus of the total matrix element is less than $1 \%$ of the $\left|\mathcal{M}^{\text {(imp. Born) }}\right|^{2}$. So, we neglect the box diagrams in the $\chi$ boson width analysis. We also found that the loop corrections $\left|\delta \Gamma_{\phi}\right| \ll 1$ and $\left|\delta \Gamma_{\chi}\right| \ll 1$ for the considered area of the model parameter space, as well as the order of their absolute values do not exceed $10^{-2}$. Due to this, the matrix element (6) can be simplified to the following form:

$$
\begin{aligned}
& i \mathcal{M}^{\text {(imp. Born) }} \approx i\left(\begin{array}{c}
g_{\phi} \\
g_{\chi}
\end{array}\right)^{T} \times \\
& \times\left(\begin{array}{cc}
p^{2}-\mu^{2}-\Pi_{\phi \phi}\left(p^{2}\right) & -\Pi_{\phi \chi}\left(p^{2}\right) \\
-\Pi_{\phi \chi}\left(p^{2}\right) & p^{2}-\Lambda^{2}-\Pi_{\chi \chi}\left(p^{2}\right)
\end{array}\right)^{-1}\left(\begin{array}{l}
g_{\phi} \\
g_{\chi}
\end{array}\right) .
\end{aligned}
$$

The matrix between the coupling constant vectors is the two-point bosonic Green function in the momentum representation. Its diagonal elements correspond to the functions $\left\langle 0\left|T \phi\left(x_{1}\right) \phi\left(x_{2}\right)\right| 0\right\rangle$ and $\left\langle 0\left|T \chi\left(x_{1}\right) \chi\left(x_{2}\right)\right| 0\right\rangle$, while the off-diagonal element corresponds to $\left\langle 0\left|T \phi\left(x_{1}\right) \chi\left(x_{2}\right)\right| 0\right\rangle$. We then express

\footnotetext{
2 Hereafter, all numerical simulation results were obtained by means of the LoopTools [13] software.
}

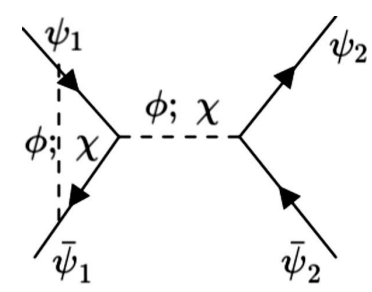

Fig. 3. General view of the loop correction to the Yukawa interaction constants $g_{\phi}$ or $g_{\chi}$. All lines and vertices are taken in the tree-approximation

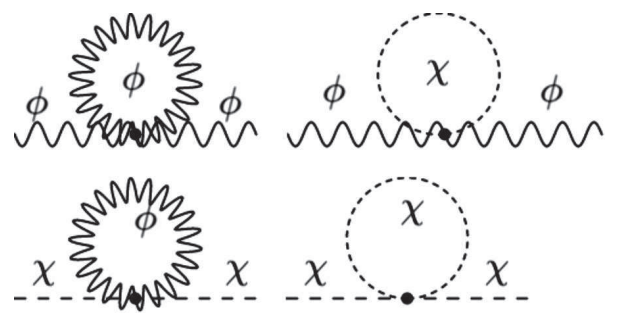

Fig. 4. Loop corrections from the self-interaction of scalar fields

(8) in terms of the fields $\phi^{\prime}$ and $\chi^{\prime}$, which are rotated by the angle $\theta_{\text {mix }}$ with respect to the $\phi$ and $\chi$, and obtain the diagonalized matrix element

$$
\begin{aligned}
& i \mathcal{M}^{\text {(diag. })}=\frac{i\left(g_{\phi} \cos \theta_{\text {mix }}+g_{\chi} \sin \theta_{\text {mix }}\right)^{2}}{\left(p^{2}-\mu^{2}-\Pi_{\phi \phi}\left(p^{2}\right)\right)\left(1-\frac{\Pi_{\chi \chi}\left(p^{2}\right)}{p^{2}-\Lambda^{2}}\right)}+ \\
& +\frac{i\left(-g_{\phi} \sin \theta_{\text {mix }}+g_{\chi} \cos \theta_{\text {mix }}\right)^{2}}{\left(p^{2}-\Lambda^{2}-\Pi_{\chi \chi}\left(p^{2}\right)\right)\left(1-\frac{\Pi_{\phi \phi}\left(p^{2}\right)}{p^{2}-\mu^{2}}\right)}+O\left(g^{4}\right) .
\end{aligned}
$$

In this expression, all contributions having higher orders in powers of the Yukawa couplings are denoted as $O\left(g^{4}\right)$. This term also depends on the external momentum. Hence, the rotation of the scalar fields by the angle $\theta_{\text {mix }}$ diagonalizes the matrix element (8) in the second order in the Yukawa couplings, whereas the non-diagonal terms are proportional to the higher powers of the couplings.

The matrix element (9) corresponds to the scattering process, where the interaction is mediated through the "physical" bosons $\phi^{\prime}$ and $\chi^{\prime}$, instead of the initial particles $\phi$ and $\chi$. That is, the resonances in the corresponding cross-section are formed out the $\phi^{\prime}$ and $\chi^{\prime}$ for $p^{2}=\mu^{2}$ and $p^{2}=\Lambda^{2}$. The first and second terms in (9) correspond to the $\phi^{\prime}$ and $\chi^{\prime}$ exchanges, respectively. Thus, the width $\Gamma$ of the $\chi^{\prime}$ resonance is determined by the imaginary part of the polarization 

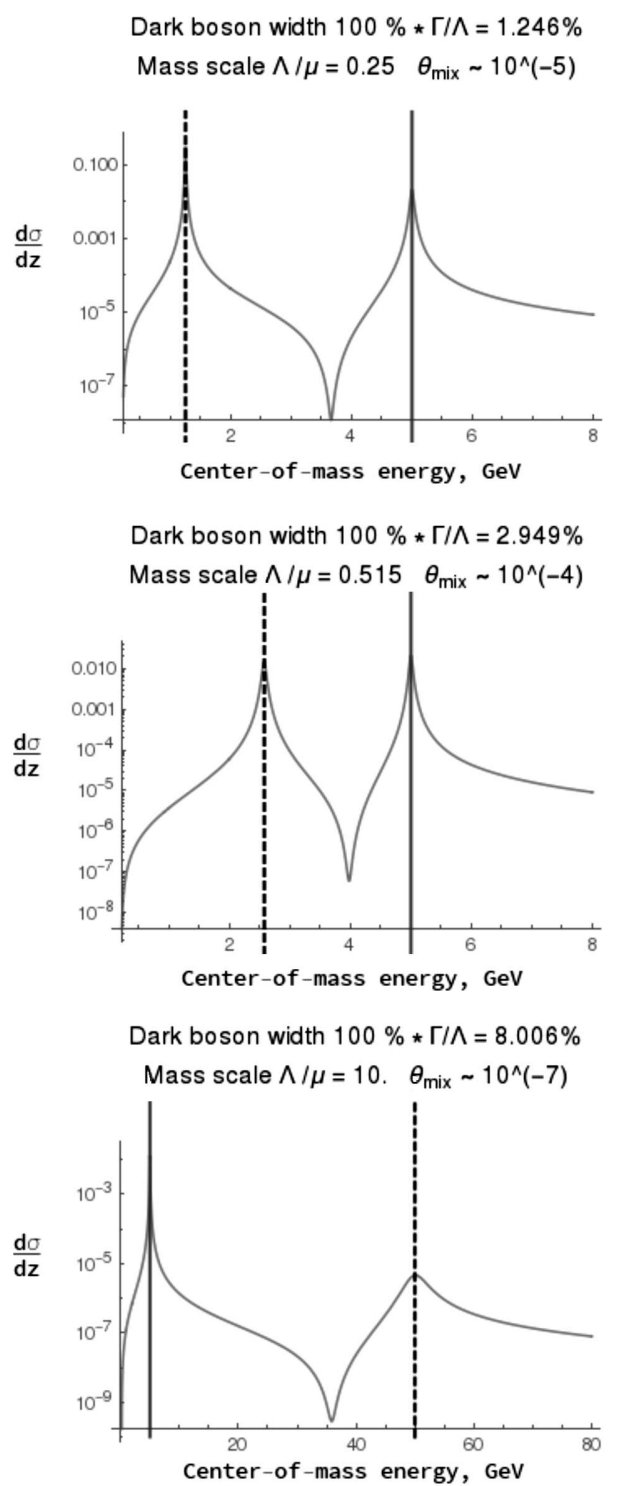

Fig. 5. Invariant mass spectra for the reaction products in Fig. 2 for various $\Lambda$. $\Lambda$ values are given as fractions of the fixed $\mu$ mass. Peak positions are denoted as solid line - visible $\phi$, dashed line - dark $\chi$

operator component $\Pi_{\chi \chi}\left(p^{2}\right)$ and reads

$$
\begin{aligned}
& \Gamma \approx \frac{\Im \Pi_{\chi \chi}\left(p^{2}=\Lambda^{2}\right)}{\Lambda}=\frac{G_{\chi}^{2} \Lambda}{8 \pi}\left(1-\frac{4 M^{2}}{\Lambda^{2}}\right)^{3 / 2}+ \\
& +\frac{g_{\chi}^{2} \Lambda}{8 \pi}\left[\left(1-\frac{4 m_{1}^{2}}{\Lambda^{2}}\right)^{3 / 2}+\left(1-\frac{4 m_{2}^{2}}{\Lambda^{2}}\right)^{3 / 2}\right]+O\left(g^{4}\right) .
\end{aligned}
$$

As free parameters, we have the dark boson mass $\Lambda$, couplings $g_{\chi}$ and $G_{\chi}$, mixing angle $\theta_{\text {mix }}$, and dark
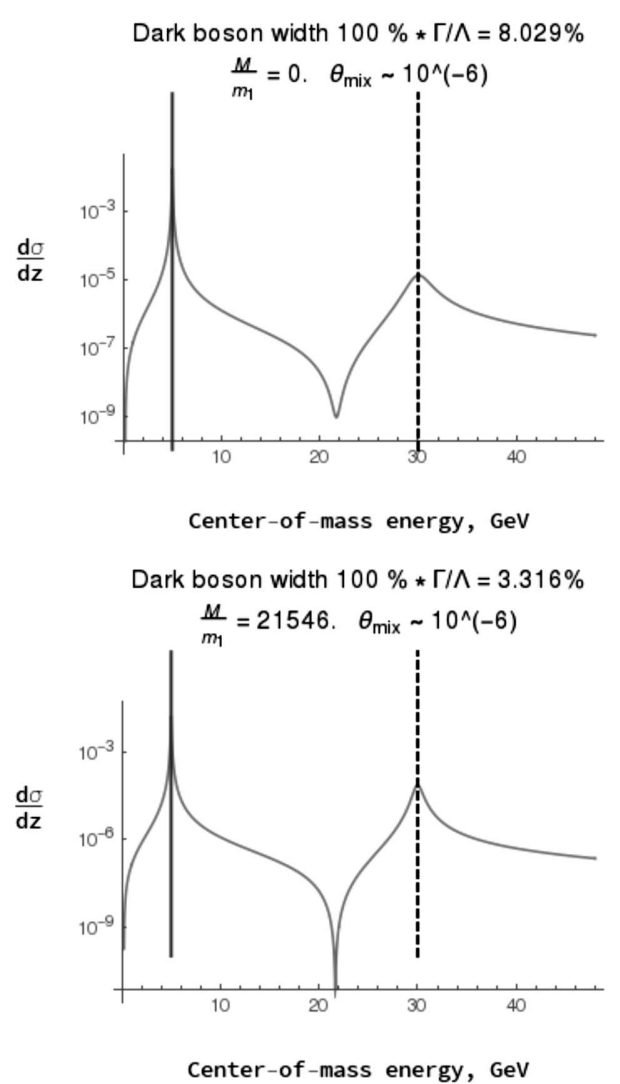

Fig. 6. Invariant mass spectra for the reaction products in Fig. 2 for various $M$. Here, $\Lambda>\mu$ and $G_{\chi} \approx 3 g_{\phi}$. Peak positions are denoted as solid line - visible $\phi$, dashed line dark $\chi$

fermion mass $M$. These quantities are the most interesting, since they contain the information about properties of the dark sector and its interaction with the visible matter. These values affect the width of the dark boson. In the model, we have such ranges of these parameters, where the resonance of $\chi$ becomes too wide to be described by the NWA. The invariant mass spectra for some values of the parameters and corresponding dark peak widths are depicted in Fig. 5, 6. In these figures, the width of a visible particle is always small and lies in the interval $\sim 1-$ $2 \%$. The vertical axis in the figures denotes the differential cross-section $\frac{d \sigma}{d z}$ value, where $z=2 \pi \cos \theta$ and $\theta$ is an angle between the directions of the initial $\psi_{1}$ and final $\psi_{2}$. The differential cross-section is given in $\mathrm{GeV}^{-2}$ in all figures of this paper.

We make conclusion about the validity of the NWA, by applying it to the DM resonances having different

ISSN 2071-0194. Ukr. J. Phys. 2021. Vol. 66, No. 11 
parameter values ${ }^{3}$. Generally, the NWA-estimated contribution $\sigma_{d}^{(\mathrm{NWA})}$ of the dark resonance into the total cross-section deviates significantly from its value $\sigma_{d}$ numerically integrated over $p^{2}$, when the $\chi$ width rises beyond $6-7 \%$. This value has to be considered as the upper width limit for the NWA applicability in our model. This bound is in addition to the 3\%limit, introduced by the experimental data treatment techniques.

The results on the dark boson width calculation are collected in Figs. 7 and 8. The widths presented there were calculated analytically, according to formula $(10)^{4}$. They are presented as the contour maps of the $\chi$ widths with two specific contours corresponding to 3 and $7 \%$ shown as black solid lines. There are areas which contain the peaks having the ratio $\rho \equiv \Gamma / \Lambda$ less than $3 \%$. So, they are potentially visible in experiments. The areas where $\rho>7 \%$ contain wide states and show the widths beyond the applicability of the NWA. The last areas in the graphs contain widths which can still be captured through the NWA, although they are wider than $3 \%$. Moreover, the values of the mixing angle are present there. In all these figures, the parameter $M$ is taken to be the same $\left(M \approx 2 \times 10^{3} m_{1}\right)$.

As we can see from the second graph in Fig. 7, if a new boson interacts sufficiently strongly with other particles inside the dark sector, the formation of new narrow resonance is rather exclusive than typical. Namely, this is so, when a new particle is lighter than the known one. Such boson is easily detectable, so this case could be rejected. For $G_{\chi} \gg g_{\phi}$, we found that if a new particle is heavier than the visible one, its peak has to be wide. Contrary to this, if $\chi$ and $\Psi$ interact weakly, the new peak is narrow in a wide interval of its mass and for the coupling $g_{\chi} \approx g_{\phi}$. In the last case, if $\Lambda \leq \mu$, the NWA is applicable to the dark peak for the almost whole $G_{\chi}$ variation interval (Fig. 8). But if the

${ }^{3}$ Detailed data behind such estimate are given in Appendix B and in Tables 12,3 , and 4 .

4 The validity of the analytic approximation (10) was assessed via the numerical calculation of the dark resonance width, from the expression for the cross-section of the process in Fig. 2. The Yukawa vertex corrections (Fig. 3) were taken into account in this calculation. It was found that the widths values from (10) differ from the numerical results by less than $2 \%$ of the mass $\Lambda$. Hence, formula (10) provides the approximation of the $\chi$ width, which is good enough in the considered intervals of model parameters.
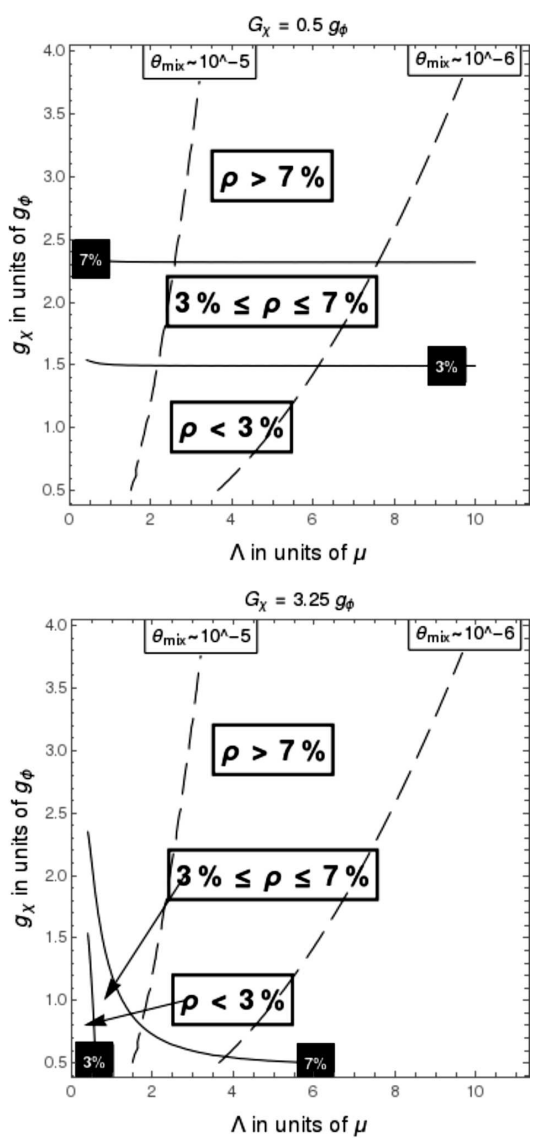

Fig. 7. Countour maps of dark boson widths and $\theta_{\operatorname{mix}}$ magnitude levels in the parametric space $\Lambda-g_{\chi}$ with fixed $G_{\chi}$. Contours corresponding to the different $\rho$ are subscribed with black labels, while the $\theta_{\text {mix }}$ magnitude levels are subscribed with white labels

coupling $g_{\chi} \gg g_{\phi}$, the detection of a dark resonance with the use of NWA is impossible. As we can see from the last graph in Fig. 8, the narrow peaks do not exist in that case. Thus, to keep the wide dark resonance, there has to be $\Lambda>\mu$ and either $g_{\chi} \gg g_{\phi}$ or $G_{\chi} \gg g_{\phi}$.

According to the results of modern experiments, new hypothetical bosons beyond the Standard model do not change the properties of known resonances [5]. It is so, if the masses of two resonances are far enough from each other, and they do not overlap. This condition is satisfied, in particular, when the mixing angle $\theta_{\text {mix }}$ is small enough. For our model, we find that the necessary condition has to be $\theta_{\text {mix }} \leq 10^{-5}$. The mixing angle levels are also shown in Figs. 7 and 8 as black dashed lines. As it is depicted in the first 

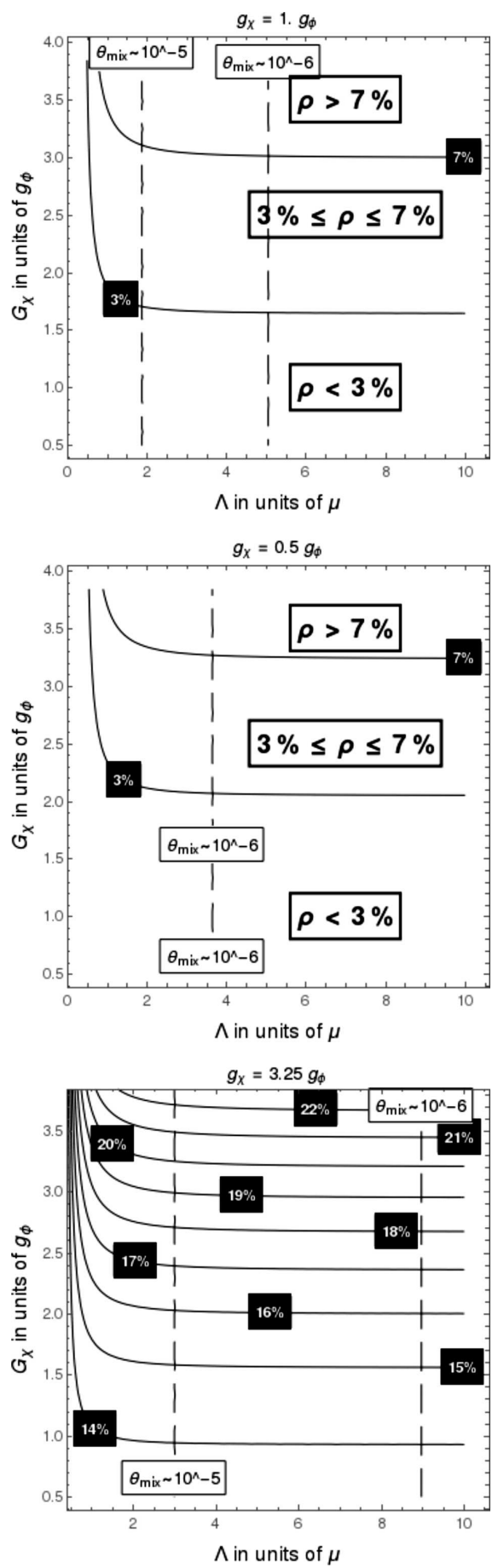

Fig. 8. Contour maps of dark boson widths and $\theta_{\text {mix }}$ magnitude levels in the parametric space $\Lambda-G_{\chi}$ with fixed $g_{\chi}$. Contours corresponding to the different $\rho$ are subscribed with black labels, while $\theta_{\text {mix }}$ magnitude levels are subscribed with white labels plot of Fig. 8, the region $\theta_{\text {mix }}>10^{-5}$ corresponds to $\Lambda=O(\mu)$. If it is so, the effects of the new physics could be detected either in the direct on-resonance searching for a narrow dark peak or through its influence on the properties of visible particles, if it is wide.

To summarize, we have investigated the influence of the dark fermion mass $M$ on the $\chi$-particle width. It is worth noting that the existence of additional fermions beyond the visible sector is not obligatory. This is because only the properties of the dark boson particle are under consideration. Nevertheless, the condition of wide $\chi$ resonance leads to certain restrictions on the $\Psi$-field mass. As we can see from the plots in Fig. 6, when the mass $M$ is increased, the DM peak is narrowing. Hence, to keep the width of $\chi$ bigger than 3\%, we anticipate dark fermions to have the mass values on the scale of visibles ones. The estimate of the $M$ upper bound is $M \leq\left(10^{3}-10^{4}\right) m_{1}$. Recall also that we have considered the contribution of light enough visible fermions to the boson widths, so that $2 m_{1 ; 2} \ll \Lambda$.

\section{Conclusions}

In the previous sections within a generalized Yukawa model, we have analyzed the role of particle coupling values and masses resulting in the creation of wide resonances in scattering processes. Such resonances could not be detected by the standard methods of direct searches under the assumption of the applicability of the NWA. To realize that, we divided the fields of the model into two classes - "visible" and "dark" ones - and have considered a number of scenarios ensuring the DM boson gains a large resonance width in the invariant mass spectrum of final states. It turns out that the limit of $3 \%$ can be exceeded in many cases. In the framework of our model, the conditions for that are the following:

- DM particle is heavier than the visible one $\Lambda>\mu$

- mixing angle $\left|\theta_{\text {mix }}\right| \leq 10^{-5}$;

- interactions in the visible sector are weaker than those between the dark and visible particles or between the particles in the dark sector only. That is, if either $g_{\chi} \gg g_{\phi}$ or $G_{\chi} \gg g_{\phi}$.

In certain cases where the width of a dark boson $\geq 7 \%$, the NWA is not applicable to the resonance. Hence, such peaks are potentially invisible in direct on-resonance searches. Moreover, if the NWA is nevertheless used for calculations of the dark resonance

ISSN 2071-0194. Ukr. J. Phys. 2021. Vol. 66, No. 11 
contribution to the total cross-section, it could result in the incorrect estimate of the particle mass and its couplings. Hence, the correct estimate of the maximal DM width is important for the processing of experimental data. This does not depend on a specific model of the DM.

In the present paper, the $s$-channel process $\psi_{1} \bar{\psi}_{1} \rightarrow$ $\rightarrow \psi_{2} \bar{\psi}_{2}$ was considered. It is similar to the process $l_{1} \bar{l}_{1} \rightarrow l_{2} \bar{l}_{2}$, where $l_{i}$ is the SM lepton of generation $i$. For example, this can be the one $e^{+} e^{-} \rightarrow \mu^{+} \mu^{-}$. In this reaction, a hypothetical $Z^{\prime}$ boson could appear as a virtual state, additionally to the SM photon and $Z$ boson. In this context, the physical meaning of the $\theta_{\text {mix }}$ limit is similar to that introduced in the phenomenology of a $Z^{\prime}$ boson. This particle is presumably mixed with the SM $Z$ boson. The corresponding mixing angle $\theta_{0}$ is experimentally bounded to the range $10^{-4}-10^{-3}$ [5]. Simultaneously, $Z^{\prime}$ has to be much heavier than $Z$ [9]. This corresponds to other condition derived above. Hence, we can conclude that the parameters of a visible particle resonance are independent of the characteristics of the dark sector. This is so until the mixing between visible and dark bosons is small, and two resonances are located far enough from each other, avoiding the interference between them. The presence of the upper limit on $\theta_{\text {mix }}$ is qualitatively important.

The considered Yukawa model gave us a possibility for analyzing the role of the masses and couplings of particles. Other aspects of the problem such as the group symmetry of the extended model and, hence, the content of the states are left behind it by us. In this approach, we have obtained the set of conditions which should be taken into consideration, when the searches for the DM particles are performed. As general conclusion, to overcome the problem of wide resonance states, it is reasonable to apply additionally some non-resonant methods to detect these new states of matter. Among them, the interference of dark and visible states should be taken into consideration at energies far from the resonance peak. Different effective Lagrangians could be derived to describe the interactions between two worlds. These are problems which we left for the future.

\section{APPENDIX A}

Consider the one-loop matrix element of the process $\psi_{1} \bar{\psi}_{1} \rightarrow$ $\rightarrow \psi_{2} \bar{\psi}_{2}$ :

$i \mathcal{M}\left(p_{1} ; p_{2} \rightarrow p_{3} ; p_{4}\right)=i \mathcal{M}^{(\text {imp. Born })}\left(p_{1} ; p_{2} \rightarrow p_{3} ; p_{4}\right)+$

ISSN 2071-0194. Ukr. J. Phys. 2021. Vol. 66, No. 11 $+i \mathcal{M}^{\text {(box })}\left(p_{1} ; p_{2} \rightarrow p_{3} ; p_{4}\right)$

In this equation, $i \mathcal{M}^{(\mathrm{imp} . \text { Born) }}$ stands for the improved Born approximation contribution, while $i \mathcal{M}^{\text {(box) }}$ comes from the box diagrams. The squared modulus of $i \mathcal{M}$ is given as follows:

$|\mathcal{M}|^{2}=\left|\mathcal{M}^{(\text {imp. Born })}\right|^{2}+$

$+2 \Re\left\{\left(\mathcal{M}^{\text {imp. Born }}\right)^{*} \mathcal{M}^{(\text {box })}\right\}+\left|\mathcal{M}^{(\text {box })}\right|^{2}$.

In the present research, we assess the difference between $|\mathcal{M}|^{2}$ and $\left|\mathcal{M}^{\text {(imp. Born) }}\right|^{2}$, omitting the term $\left|\mathcal{M}^{(\text {box })}\right|^{2}$. We do so because of the technical difficulties in the calculation of $\left|\mathcal{M}^{(\text {box })}\right|^{2}$. Hence, $\left|\mathcal{M}^{(\text {imp. Born })}\right|^{2}$ is compared with the fol-

Table 1. $\mathbf{\Lambda}$ variation

\begin{tabular}{|l|c|c|c|}
\hline$\rho, \%$ & $\Lambda$ & $\frac{\left|\sigma_{v}-\sigma_{v}^{(\mathrm{NWA})}\right|}{\sigma_{v}}, \%$ & $\frac{\left|\sigma_{d}-\sigma_{d}^{(\mathrm{NWA})}\right|}{\sigma_{d}}, \%$ \\
\hline 1.26 & $0.25 \mu$ & 4.839 & 2.077 \\
6.3 & $0.9 \mu$ & 4.955 & 5.63 \\
7.867 & $3 \mu$ & 4.232 & 5.898 \\
7.983 & $6 \mu$ & 3.468 & 5.921 \\
\hline
\end{tabular}

Table 2. $M$ variation

\begin{tabular}{|l|c|c|c|}
\hline \multicolumn{1}{|c|}{$\%$} & $M$ & $\frac{\left|\sigma_{v}-\sigma_{v}^{(\mathrm{NWA})}\right|}{\sigma_{v}}, \%$ & $\frac{\left|\sigma_{d}-\sigma_{d}^{(\mathrm{NWA})}\right|}{\sigma_{d}}, \%$ \\
\hline 8.033 & 0 & 3.468 & 6.004 \\
8.1 & $m_{1}$ & 3.468 & 7.703 \\
7.983 & $\approx 2000 m_{1}$ & 3.468 & 5.921 \\
\hline
\end{tabular}

Table 3. $\boldsymbol{g}_{\chi}$ variation

\begin{tabular}{|c|c|c|c|}
\hline$\rho, \%$ & $g_{\chi}$ & $\frac{\left|\sigma_{v}-\sigma_{v}^{(\mathrm{NWA})}\right|}{\sigma_{v}}, \%$ & $\frac{\left|\sigma_{d}-\sigma_{d}^{(\mathrm{NWA})}\right|}{\sigma_{d}}, \%$ \\
\hline 7.975 & $1 . g_{\phi}$ & 3.468 & 5.921 \\
13.81 & $2.438 g_{\phi}$ & 3.678 & 9.283 \\
19.41 & $3.25 g_{\phi}$ & 3.856 & 14.81 \\
26.23 & $4 . g_{\phi}$ & 3.945 & 22.38 \\
56.95 & $5.9 g_{\phi}$ & 3.353 & 51.94 \\
\hline
\end{tabular}

Table 4. $\boldsymbol{G}_{\chi}$ variation

\begin{tabular}{|c|c|c|c|}
\hline$\rho, \%$ & $G_{\chi}$ & $\frac{\left|\sigma_{v}-\sigma_{v}^{(\mathrm{NWA})}\right|}{\sigma_{v}}, \%$ & $\frac{\left|\sigma_{d}-\sigma_{d}^{(\mathrm{NWA})}\right|}{\sigma_{d}}, \%$ \\
\hline 1.231 & $0.01 g_{\phi}$ & 3.482 & 2.429 \\
1.378 & $0.5 g_{\phi}$ & 3.482 & 1.59 \\
3.765 & $2 . g_{\phi}$ & 3.483 & 3.259 \\
7.99 & $3.25 g_{\phi}$ & 3.484 & 5.955 \\
17.78 & $5 . g_{\phi}$ & 3.485 & 11.26 \\
40.96 & $7.5 g_{\phi}$ & 3.489 & 15.91 \\
\hline
\end{tabular}


lowing approximation:

$|\mathcal{M}|^{2} \approx\left|\mathcal{M}^{(\text {imp. Born })}\right|^{2}+2 \Re\left\{\left(\mathcal{M}^{\text {imp. Born }}\right)^{*} \mathcal{M}^{(\text {box })}\right\}$.

We found that, for various intervals of the model parameters, $|\mathcal{M}|^{2}$ differs from $\left|\mathcal{M}^{\text {(imp. Born) }}\right|^{2}$ by the fraction less than $1 \%$ of $\left|\mathcal{M}^{\text {(imp. Born) }}\right|^{2}$. Thus, the $\left|\mathcal{M}^{\text {(box) }}\right|^{2}$ contribution would be even less, and the neglection of this term is valid. Eventually, we ignore the contribution of box diagrams $\mathcal{M}^{\text {(box) }}$ as a whole, since its effect on particles' widths is negligibly small.

\section{APPENDIX B}

The error of the NWA is estimated as a deviation of $\sigma_{d}$ from $\sigma_{d}^{(\text {NWA })}$ as a fraction of $\sigma_{d}$. We consider such error as insignificant, if its absolute value is less than or equal to the corresponding deviation for a visible resonance described by the contribution $\sigma_{v}$ and its approximation $\sigma_{v}^{(\mathrm{NWA})}$. The estimations were performed for the different model parameters (see Tables 1-4).

1. Sunghoon Jung, Dongsub Lee, Ke-Pan Xie. Beyond $M_{t \bar{t}}$ : learning to search for a broad $t \bar{t}$ resonance at the LHC. Eur. Phys. J. C 80, 105 (2020).

2. S. Moretti, D. O'Brien, L. Panizzi and Hugo Prager. Production of extra quarks decaying to dark matter beyond the narrow width approximation at the LHC. Phys. Rev. D 96 (3), 035033 (2017).

3. S. Moretti, D. O'Brien, L. Panizzi, H. Prager. Production of extra quarks at the Large Hadron Collider beyond the narrow width approximation. Phys. Rev. D 96 (7), 075035 (2017).

4. M. Drees and F. Hajkarim. Neutralino dark matter in scenarios with early matter domination. JHEP 2018, 42 (2018).

5. A. Gulov, V. Skalozub. Global Search For The Z' Boson In Scattering Processes. (Lira, 2018) [ISBN: 978-966-981143-1].

6. P. Langacker. The physics of heavy Z' gauge bosons. ePrint: 0801.1345v3 [hep-ph] (2009).

7. A. Leike. The phenomenology of extra neutral gauge bosons. e-Print: hep-ph/9805494v1 (1999).

8. A. Boyarsky, M. Drewes, T. Lasserre, S. Mertens, O. Ruchayskiy. Sterile neutrino dark matter. e-Print: 1807.07938 [hep-ph] (2018).
9. M. Tanabashi et al. (Particle Data Group). Review of particle physics. Phys. Rev. D 98, 030001 (2018-2019).

10. Tomohiro Abe, Ryuichiro Kitano, Ryosuke Sato. Discrimination of dark matter models in future experiments. Phys. Rev. D 91 (9), 095004 (2015).

11. Sunghoon Jung, Jeonghyeon Song, Yeo Woong Yoon. Dip or nothingness of a Higgs resonance from the interference with a complex phase. Phys. Rev. D 92 (5), 055009 (2015).

12. E. Fuchs, S. Thewes, G. Weiglein. Interference effects in BSM processes with a generalised narrow-width approximation. Eur. Phys. J. C 75, 253 (2015).

13. T. Hahn, M. Perez-Victoria. Automatized one-loop calculations in 4 and D dimensions. Comput. Phys. Commun 118 (2-3), 153 (1999).

Received 20.04.21

\section{В.В. Скалозуб, М.С. Дмитрієе}

ДО ПРЯМИХ ПОШУКІВ

ТЕМНОÏ МАТЕРIÏ У ПРОЦЕСАХ

РОЗСІЮВАННЯ В МОДЕЛІ ЮКАВИ

До теперішнього часу не було виявлено жодної частинкикандидата на роль темної матерї. Ми розглядаємо дві можливі причини цього, які пов'язані із методом on-peak пошуку резонансів. Як зазвичай припускається, ширина резонансу нової частинки менша, ніж 1-3\% від їі маси, і для пошуку цього резонансу у спектрі інваріантної маси продуктів зіткнення можна використовувати наближення вузького піка (narrow width approximation, NWA). У цій статті на прикладі узагальненої моделі Юкави ми отримуємо властивості шуканої частинки, за яких ширина їі резонансу є більшою, ніж та, що максимально припустима для застосування NWA в аналізі результатів експерименту. В такому випадку цей стан може бути невірно трактований як шум. Ми також отримуємо значення параметрів частинки та ширини її резонансу, за яких NWA не може бути застосований. Ці оцінки актуальні також для взаємодії між частинками Стандартної моделі та темної матеріі. У застосованому підході увага зосереджена на ролі величин констант зв'язку та мас, що вводяться у моделі взаємодії видимої та темної матерії.

Kлючовi слова: темна матерія, резонанс, наближення вузького піка, змішування полів. 